\title{
Paper
}

\section{Optimization of Bonding and Sizing Process for Automotive Wet Clutch Discs applying "Robust Design"}

\author{
Eiji Toma ${ }^{* \dagger}$ Member, Katsumi MiYama ${ }^{*}$ Non-member
}

(Received December 12, 2019, revised April 15, 2020)

\begin{abstract}
Many parts for transmitting power are incorporated in an automatic transmission of an automobile. The technical problems of the wet clutch disc (DISC) having important functions related to gear shifting are to improve the automatic gear shifting performance and to ensure the stability of the power transmission torque in the contact action between the DISCs. DISC is manufactured by adhesive bonding of friction material $(\mathrm{Fa})$ and iron core plate $(\mathrm{C} / \mathrm{P})$. In particular, the stabilization of the DISC adhesive strength and thickness accuracy suppresses fluctuations in the surface friction coefficient and is effective in reducing the shock phenomenon during shifting. In this study, the robustness of the DISC bonding sizing method by applying robust design was aimed at stabilizing the bonding strength and thickness accuracy, and the optimum processing conditions were extracted to optimize the mass production method.
\end{abstract}

Keywords: Wet clutch disc, Transmission performance, Robust design, Functionality evaluation, Energetic S/N ratio,

\section{Introduction}

As shown in Fig. 1, many parts for transmitting power are incorporated in the automatic transmission of automobiles. The technical problem of the wet clutch disk (DISC), which has important functions related to gear shifting, is to improve the automatic gear shifting performance and to make the power transmission torque robust in the contact action between the DISCs [[1] [4]. DISC is manufactured by adhesive bonding of a friction material $(\mathrm{Fa})$ impregnated with a synthetic resin in special raw paper and a core plate $(\mathrm{C} / \mathrm{P})$ that is an iron material. In the field of tribology technology, stabilizing the adhesive strength and thickness accuracy of DISC is extremely effective in reducing the rate of change of the dynamic friction coefficient $(\mu)$ on the surface and increasing the robustness of the power transmission torque [5] [7]. In particular, stabilization of the DISC plate thickness accuracy after bonding has been reported to be effective in reducing fluctuations in the surface friction coefficient and reducing shock phenomena during shifting [8] [9].

In this study, we examined the mechanism of adhesive penetration during bonding and approached the optimization of the bonding sizing (plate thickness uniformity) method by verifying the correlation between the bonding strength and the plate thickness accuracy using a robust design.

\footnotetext{
* Corresponding: toma-e@ tomakomai-ct.ac.jp

$\dagger$ National Institute of Technology, Tomakomai College

443, Tomakomai, Nishikioka, Hokkaido, Japan 059-1275

$\ddagger$ Hokkaido University of Science

15-4-1, Maeda 7-jo, Sapporo-shi, Hokkaido, Japan 006-8585
}

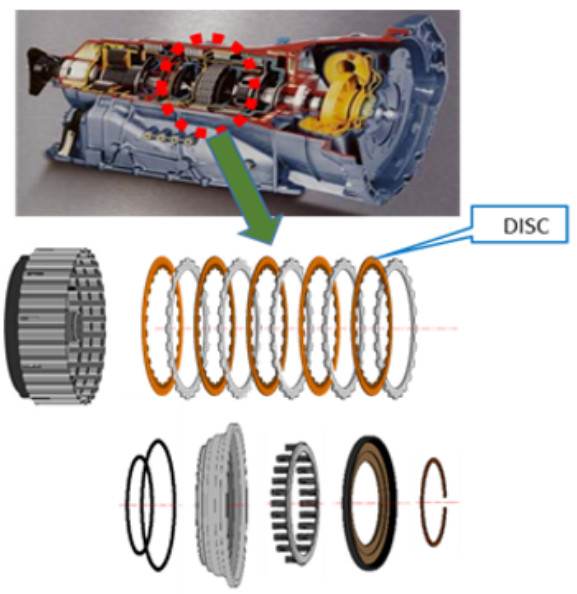

Figure 1: Components in clutch pack.

\section{Outline of bonding sizing method}

In the DISC production process, in the pre-bonding process, $\mathrm{Fa}$ and $\mathrm{C} / \mathrm{P}$ are temporarily attached by applying an adhesive, followed by a drying process for the purpose of volatilization of the solvent and water classification contained in the adhesive. As shown in Fig. 2, a hydraulic hot plate press type adhesive sizing method is adopted to ensure both DISC adhesive strength and plate thickness uniformity. The DISC after provisional application placed in the pressurization/heating station is lowered and pressurized (press load: $400 \mathrm{kN}$ ) by the cylinder thrust by hydraulic control At the same time, heating with a hot plate heater for a certain time (mold surface temperature; $250^{\circ} \mathrm{C} . \pm 10$ ). The temperature of the hot plate heater is controlled by PID control 


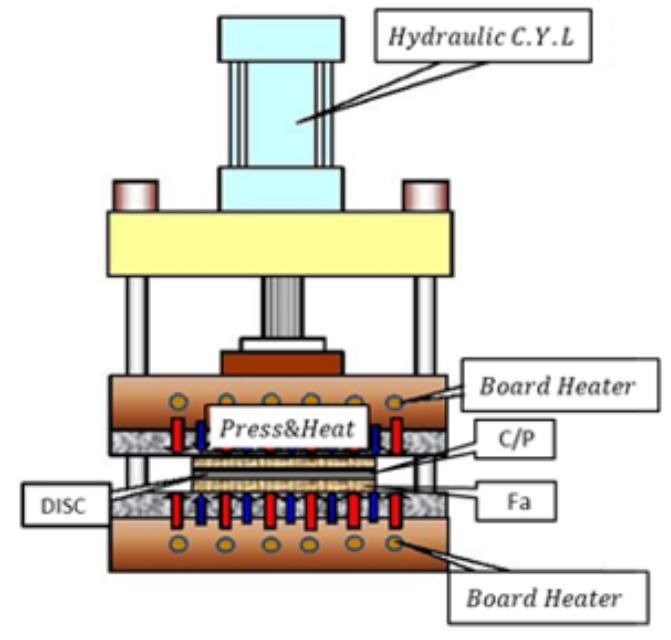

Figure 2: Bonding and Sizing process.

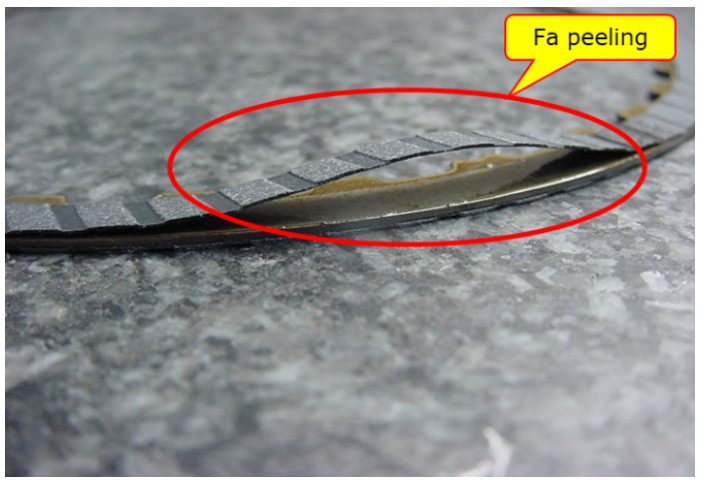

Figure 3: Adhesion failure phenomenon due to Fa peeling.

using an embedded heater and a thermocouple. Moreover, the side surface of the hot plate mold has a heat insulating structure for the purpose of increasing thermal efficiency.

\section{Quality problem and inference of factors}

3.1 Extract quality problem As shown in Fig. 3, Fa peeling occurs in part after bonding sizing, and there is a quality problem of defective bonding. Fa peeling significantly deteriorates the automatic transmission performance which is an important function of the clutch disk [10]. Moreover, it will lead to the occurrence of a serious failure related to the safe driving of the automobile.

3.2 Inference of factors Figure 4 is a photograph showing the Fa peeling phenomenon in detail. In Fig. 4(a), the Fa peeled part occurred in one direction (facing direction) of the DISC, and the expansion displacement of the DISC due to pressure and heating during bonding was observed. In Fig. 4(b), almost no adhesive remained in the peeled portion on the $\mathrm{C} / \mathrm{P}$ side, and it was observed that the adhesive penetrated into the peeled $\mathrm{Fa}$.

As shown in Fig. 5, in the drying process after the adhesive application and tacking process, the solvent and moisture contained in the adhesive remain due to insufficient drying, and they penetrate into $\mathrm{Fa}$. The non-uniformity in the amount of adhesive applied is presumed to be a Fa peel-

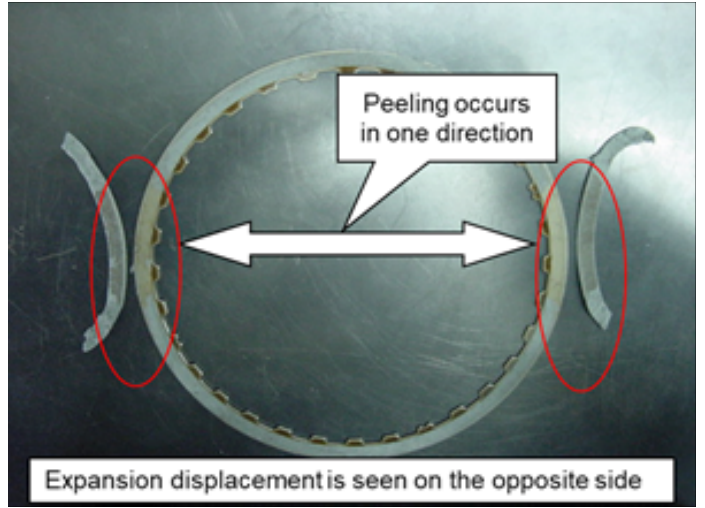

(a) Fa peeling location

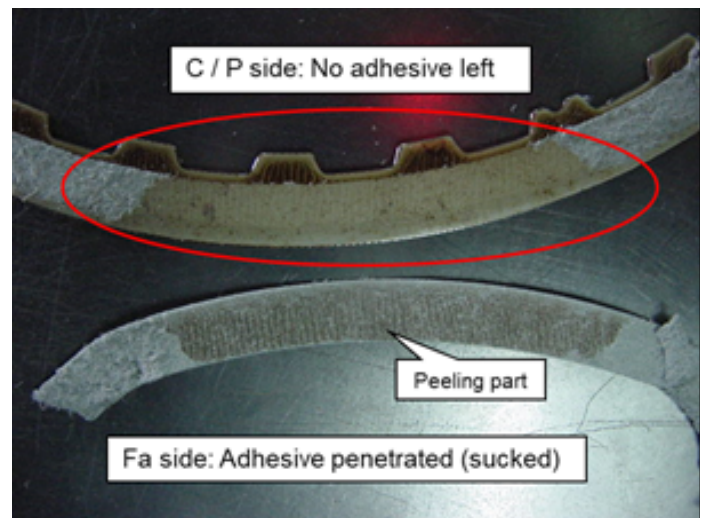

(b) Application state of adhesive

Figure 4: Verification/estimation of adhesion failure mode.

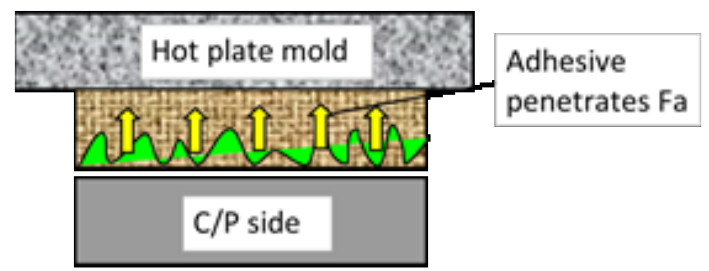

Figure 5: Adhesive unevenness mechanism.

ing factor.

\section{Application of "Robust design"}

In the previous chapter, we extracted the quality problems of DISC adhesion failure due to Fa peeling and made inferences about the factor system. In this chapter, we approached the stabilization of DISC adhesive strength and plate thickness accuracy of the adhesive sizing method by applying the functional evaluation method of parameter design, which is the central method of "Robust design".

4.1 Overview of Robust design Figure 6 shows the concept of robust design in quality engineering. The concept of improving technology to bring it closer to what it should be is called robust design. Robust means stability in quality engineering. Parameter design is one of the central methods of robust design, and is a method for evaluating functionality and determining system parameter values. Parameters are design constants and components of the sys- 
tem and are selected as control factors in parameter design experiments. In the combination of system parameters, robustness is improved by deliberately generating variations by noise factors and optimizing the level of strong control factors that can counter the variations [11] [13].

In parameter design, it is important to understand the target function of the system. The objective function refers to the role that the system should play, and the quality characteristics are divided into the following four types. By taking up the objective function, the design information can be utilized in other similar systems, and the versatility of the technology is expanded [14] [15].

(a) Preferably small characteristics (nonnegative and smaller the better)

(b) Preferably large characteristics (nonnegative and larger the better)

(c) Preferably target characteristics (with target value)

(d) Dynamic characteristics (I/O correlation, output stability)

In this study, stabilization of DISC adhesive strength and plate thickness accuracy in the adhesive sizing method is the objective function, and the optimum parameter level condition for obtaining it is extracted by dynamic characteristic evaluation.

The evaluation procedure for robust design is shown in Fig. 7. In robust design, start by clarifying the functions of the system. We define it as an ideal function of the input (signal factor) and output (characteristic value) of the system. In order to realize the ideal function, the experiment is performed efficiently using the orthogonal table, and the effect of each control factor is grasped by the SN ratio, sensitivity, and slope $\beta$. After that, the optimum conditions and comparison conditions are determined from the factor-effect diagram, and the average of each process is obtained to estimate the magnitude of improvement. Finally, a confirmation experiment is performed to verify the reproducibility of the optimum condition gain.

4.2 Definition of ideal function The ideal function of many system technologies is that the output varies linearly with respect to the input. The total output characteristic value of the system (total work) must be proportional to energy or work. Especially in the bonding sizing process of DISC, there are input energy (bonding time, etc.) necessary to obtain bonding strength and energy ( $\mathrm{C} / \mathrm{P}$ surface temperature, etc.) that affects bonding time. In robust

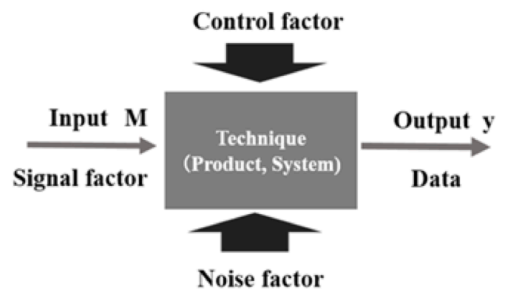

Figure 6: Robust design. design, a "Functional evaluation method" that defines functionality based on energy conversion and quickly evaluates its stability is important [16].

In this study, we focused on the fact that the ideal function representing the relationship between input and output becomes linear with energy conversion, and defined an ideal functional state as shown in Fig. 8. The main purpose of the "Functional evaluation method" is to efficiently extract parameters that reduce variations and fluctuations by bringing the stable function of adhesive strength and plate thickness accuracy closer to the ideal state. The difference from the ideal state can be considered as some energy loss.

4.3 Energetic $\mathrm{S} / \mathrm{N}$ ratio The conventional $\mathrm{SN}$ ratio (Signal-to-Noise ratio) used in parameter design, which represents a measure of variation, may change depending on the size (range) of the input signal and the number of data. There is a problem that engineers must acquire and analyze data while keeping this in mind. The idea of the SN ratio is to evaluate the dispersion and non-linearity from the ideal function state $(y=\beta M)$ by considering the functional stability to be poor. The "Energetic S/N ratio" adopted in this study is a new $\mathrm{SN}$ ratio that was researched by a research member of the Kansai Quality Engineering Society, and was announced in June 2008 [17].

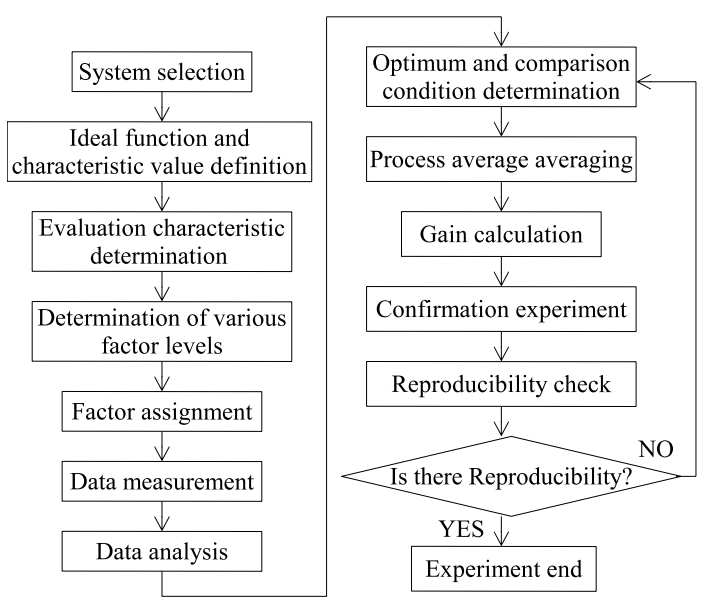

Figure 7: Robust design procedure.

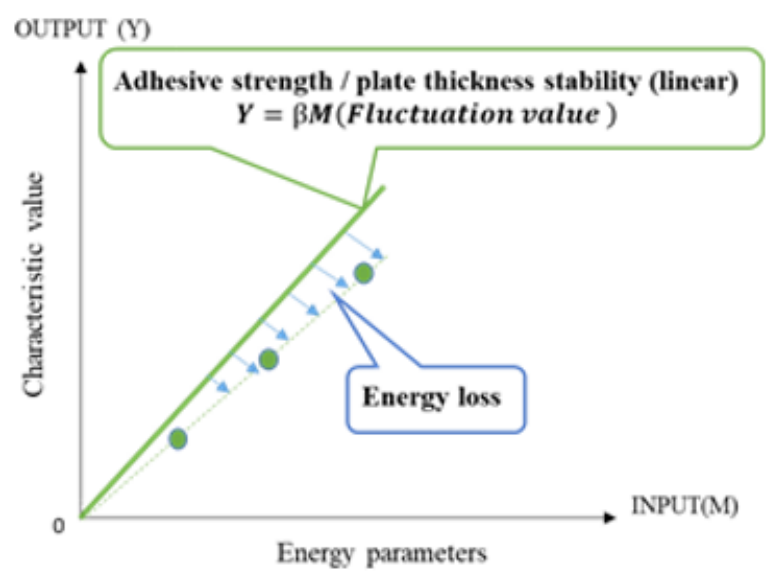

Figure 8: Definition of ideal function . 
Table 1: Correspondence table for SN ratio calculation.

\begin{tabular}{|c|c|c|c|c|c|c|}
\hline \multicolumn{2}{|c|}{} & \multicolumn{5}{|c|}{ Input signal M } \\
\cline { 3 - 7 } & $M_{1}$ & $M_{2}$ & $M_{3}$ & $\cdots$ & $M_{k}$ \\
\hline \multirow{2}{*}{$\begin{array}{c}\text { Noise } \\
\text { factor } \\
\mathrm{N}\end{array}$} & $N_{1}$ & $y_{11}$ & $y_{12}$ & $y_{13}$ & $\cdots$ & $y_{1 k}$ \\
\cline { 2 - 7 } & $N_{2}$ & $y_{21}$ & $y_{22}$ & $y_{23}$ & $\cdots$ & $y_{2 k}$ \\
\cline { 2 - 7 } & $\vdots$ & $\vdots$ & $\vdots$ & $\vdots$ & $\vdots$ & $\vdots$ \\
\cline { 2 - 7 } & $N_{n}$ & $y_{n 1}$ & $y_{n 2}$ & $y_{n 3}$ & $\cdots$ & $y_{n k}$ \\
\hline
\end{tabular}

Since all technologies involve energy conversion and transmission, an energy perspective is important in technology development and level evaluation. The energetic $\mathrm{S} / \mathrm{N}$ ratio is a technical quality evaluation scale based on how various types of energy are used. The technical quality here means whether more energy input to the system can be stably used for the purpose (output) indefinitely.

In the energetic $\mathrm{S} / \mathrm{N}$ ratio, the energy is decomposed into an active component $\left(S_{\beta}\right)$ and a harmful component $\left(S_{N}\right)$, and is calculated from these ratios. When using $\mathrm{SN}$ ratio in parameter design, it is expressed in decibel values [db] as 10 times the common logarithm. The following is the basic formula for energetic $\mathrm{S} / \mathrm{N}$ ratio $\left(\eta_{E}\right)$.

As shown in Table 1, noise factor $n$ level, signal factor $k$ level, noise factor level $i(i=1,2, \cdots, n)$, signal factor level $j(j=1,2, \cdots, k)$, suppose that the output $y_{i j}$ is obtained.

- Total variation component $S_{T}$

This is the sum of the squares of $n k$ pieces of data $y_{i j}$ and shows the variation from $y=0$.

$$
S_{T}=\sum_{i=1}^{n} \sum_{j=1}^{k} y_{i j}^{2}
$$

\section{- Average slope size $\beta_{N 0}$}

For some dynamic characteristics of the input signal, the slope of the noise factor $N_{i}$ level $\beta_{N i}$ is obtained by considering the effective component as the average slope.

$$
\beta_{N i}=\frac{\sum_{j=1}^{k} M_{J} y_{i j}}{\sum_{j=1}^{k} M_{j}^{2}}=\frac{L_{N i}}{r}
$$

Here,

$r$ : Effective divisor, $L_{N i}$ : Linear format

$$
\begin{aligned}
r & \equiv \sum_{j=1}^{k} M_{j}^{2} \\
L_{N i} & \equiv \sum_{j=1}^{k} M_{j} y_{j} \\
\therefore \beta_{N 0} & =\frac{\sum_{i=1}^{n} \beta_{N i}}{n}
\end{aligned}
$$

- Average slope fluctuation (active ingredient) $S_{\beta}$
The variation of the average slope is expressed as the sum of squares of the size $y=\beta_{N 0} M$.

$$
\begin{aligned}
\therefore S_{\beta}=n \sum_{j=1}^{k}\left(\beta_{N 0} M_{j}\right)^{2} & =n \beta_{N 0}^{2} \sum_{j=1}^{k}\left(M_{j}\right)^{2} \\
& =n r \beta_{N 0}^{2}
\end{aligned}
$$

- Harmful component $S_{N}$

The harmful component represents the variation of the difference between each data and the proportional equation of the average slope, and is obtained by subtracting the effective component from the total variation component.

$$
\therefore S_{N}=\sum_{i=1}^{n} \sum_{j=1}^{k}\left(y_{i j}-\beta_{N 0} M_{j}\right)^{2}=S_{T}-S_{\beta}
$$

- $\mathrm{SN}$ ratio $\eta_{E}$

$$
\eta_{E}=10 \log \left(\frac{S_{\beta}}{S_{T}-S_{\beta}}\right)=10 \log \left(\frac{S_{\beta}}{S_{N}}\right)(d b)
$$

4.4 Analysis by "Dynamic characteristic" It was verified by "Dynamic characteristic evaluation (energetic $\mathrm{S} / \mathrm{N}$ ratio)" with the objective function of stabilizing the DISC adhesive strength and plate thickness accuracy in the adhesive sizing method.

Dynamic characteristic is a characteristic that examines the output by changing the input. It is a method to evaluate the condition that minimizes the two-dimensional variation of input and output among various combinations of factors. Even under various usage conditions and environments, it is desirable that the input and output signals are in a proportional relationship, and a more robust improvement effect can be obtained in terms of reproducibility, precedence, and versatility.

4.5 Determination of level tables and factors Table 2 shows the control factor level table in this experiment. As control factors, seven parameters were extracted: [Drying method], [Press load], [Heater capacity], [Adhesive coating thickness], [DISC transport speed], [Drying temperature], and [Dry hot air pressure]. Table 3 shows the level table

Table 2: Control factors.

\begin{tabular}{cccccc}
\hline \multirow{2}{*}{ Symbol } & \multirow{2}{*}{ Parameter } & Unit & \multicolumn{3}{c}{ Level } \\
\cline { 3 - 6 } & & 1 & 2 & 3 \\
\hline A & Drying method & {$[$ Pass $]$} & 2 & 1 & - \\
B & Press load & {$\left[\times 10^{2} \mathrm{kN}\right]$} & 2.2 & 3.2 & 4.2 \\
C & Heater capacity & {$[\mathrm{kw}]$} & 6.5 & 7.5 & 8.5 \\
D & Coating thickness & {$[\mu \mathrm{m}]$} & 7 & 9 & 11 \\
E & Transport speed & {$[\mathrm{m} / \mathrm{min}]$} & 8 & 10 & 12 \\
F & Drying temperature & {$\left[{ }^{\circ} \mathrm{C}\right]$} & 100 & 120 & 140 \\
G & Hot air pressure & {$\left[\mathrm{kgf} / \mathrm{cm}^{2}\right]$} & 1.5 & 2.5 & 3.5 \\
H & Virtual factor & {$[-]$} & $\mathrm{e} 1$ & $\mathrm{e} 2$ & $\mathrm{e} 3$ \\
\hline
\end{tabular}




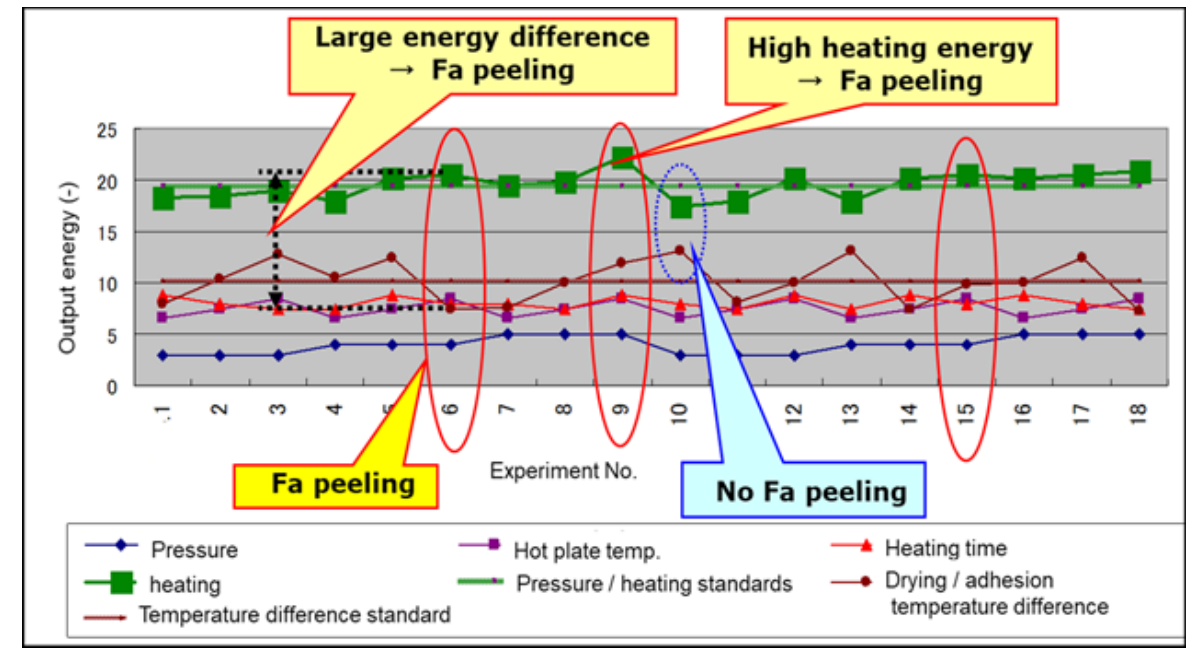

Figure 9: Adhesive energy characteristics for each experiment.

Table 3: Noise factors.

\begin{tabular}{cccc}
\hline & Unit & \multicolumn{2}{c}{ Noise factors } \\
\hline C/P surface & & $\mathrm{N} 1$ & $\mathrm{~N} 2$ \\
temperature & {$\left[{ }^{\circ} \mathrm{C}\right]$} & $23 \pm 3$ & $0 \pm 3$ \\
\hline
\end{tabular}

with the $\mathrm{C} / \mathrm{P}$ surface temperature $\left(\mathrm{N} 1 ; 23 \pm 3{ }^{\circ} \mathrm{C}, \mathrm{N} 2 ; 0 \pm 3\right.$ ${ }^{\circ} \mathrm{C}$ ) before tacking as noise factor.

Table 4 shows the L18 orthogonal data table of this experiment, and the adhesion time (M1; 4.0 [second], M2; 5.0 [second], M3; 6.0 [second]) was set as the input signal factor. The measurement data, which is the output characteristic value, is a plate thickness stability factor [dimensionless unit] obtained by calculating the reciprocal of the plate thickness difference average value at 6 locations per DISC. The purpose of setting the output characteristic value is to evaluate the stability of the adhesive strength and the plate thickness accuracy as the plate thickness difference is smaller and the plate thickness stability rate is increased.

\subsection{Consideration of adhesive energy characteristics} Figure 9 shows the results of the discussion on the relative relationship between Fa peeling and adhesion energy characteristics from L18 orthogonal table experimental results. The horizontal axis of the graph is the experiment number, and the vertical axis represents the characteristic value based on the load energy related to adhesion. From the graph, it can be considered that the expansion of $\mathrm{C} / \mathrm{P}$ and the penetration of the adhesive into $\mathrm{Fa}$ are promoted and Fa peeling occurs under the condition that the pressure and heating energy related to adhesion is large and the drying energy is insufficient.

Figure 10 shows the input/output relationship between

Table 4: L18 orthogonal table/experimental data.

\begin{tabular}{cccccccccccccccc}
\hline $\begin{array}{c}\text { EXP. } \\
\text { N0. }\end{array}$ & A & B & C & D & E & F & G & H & N1 & N2 & N1 & N2 & N1 & N2 & peeling \\
\hline 1 & 2 & 2.2 & 6.5 & 7 & 8 & 100 & 1.5 & e1 & 20.00 & 20.83 & 16.95 & 14.71 & 13.70 & 14.93 & \\
2 & 2 & 2.2 & 7.5 & 9 & 10 & 120 & 2.5 & e2 & 8.20 & 9.43 & 6.80 & 8.00 & 10.99 & 10.53 & \\
3 & 2 & 2.2 & 8.5 & 11 & 12 & 140 & 3.5 & e3 & 5.03 & 5.49 & 5.13 & 5.29 & 5.52 & 4.93 & \\
4 & 2 & 3.2 & 6.5 & 7 & 10 & 120 & 3.5 & e3 & 18.18 & 20.00 & 16.67 & 15.38 & 14.08 & 14.71 & \\
5 & 2 & 3.2 & 7.5 & 9 & 12 & 140 & 1.5 & e1 & 13.70 & 14.71 & 13.33 & 12.20 & 11.63 & 12.50 & $\sim$ \\
6 & 2 & 3.2 & 8.5 & 11 & 8 & 100 & 2.5 & e2 & 4.00 & 3.65 & 3.30 & 4.20 & 3.52 & 3.77 & $\sim$ \\
7 & 2 & 4.2 & 6.5 & 9 & 8 & 140 & 2.5 & e3 & 17.86 & 19.61 & 16.67 & 14.93 & 13.89 & 14.49 & \\
8 & 2 & 4.2 & 7.5 & 11 & 10 & 100 & 3.5 & e1 & 5.41 & 5.21 & 4.78 & 4.83 & 3.34 & 3.68 & \\
9 & 2 & 4.2 & 8.5 & 7 & 12 & 120 & 1.5 & e2 & 3.82 & 3.69 & 3.14 & 4.18 & 3.50 & 3.62 & $\sim$ \\
10 & 1 & 2.2 & 6.5 & 11 & 12 & 120 & 2.5 & e1 & 5.85 & 5.65 & 4.78 & 5.41 & 3.82 & 4.22 & \\
11 & 1 & 2.2 & 7.5 & 7 & 8 & 140 & 3.5 & e2 & 12.35 & 11.63 & 14.08 & 12.66 & 9.90 & 11.24 & \\
12 & 1 & 2.2 & 8.5 & 9 & 10 & 100 & 1.5 & e3 & 6.67 & 5.88 & 6.21 & 7.52 & 5.15 & 5.56 & \\
13 & 1 & 3.2 & 6.5 & 9 & 12 & 100 & 3.5 & e2 & 6.85 & 6.45 & 6.71 & 7.14 & 6.13 & 5.62 & \\
14 & 1 & 3.2 & 7.5 & 11 & 8 & 120 & 1.5 & e3 & 6.45 & 6.85 & 6.25 & 6.49 & 7.52 & 6.06 & $\sim$ \\
15 & 1 & 3.2 & 8.5 & 7 & 10 & 140 & 2.5 & e1 & 4.24 & 3.91 & 4.48 & 3.86 & 4.03 & 3.41 & $\sim$ \\
16 & 1 & 4.2 & 6.5 & 11 & 10 & 140 & 1.5 & e2 & 7.30 & 6.85 & 7.09 & 6.62 & 6.41 & 5.65 & \\
17 & 1 & 4.2 & 7.5 & 7 & 12 & 100 & 2.5 & e3 & 8.20 & 8.70 & 6.99 & 8.33 & 11.24 & 8.77 & \\
18 & 1 & 4.2 & 8.5 & 9 & 8 & 120 & 3.5 & e1 & 2.90 & 2.34 & 3.10 & 2.23 & 3.56 & 2.94 & \\
\hline
\end{tabular}




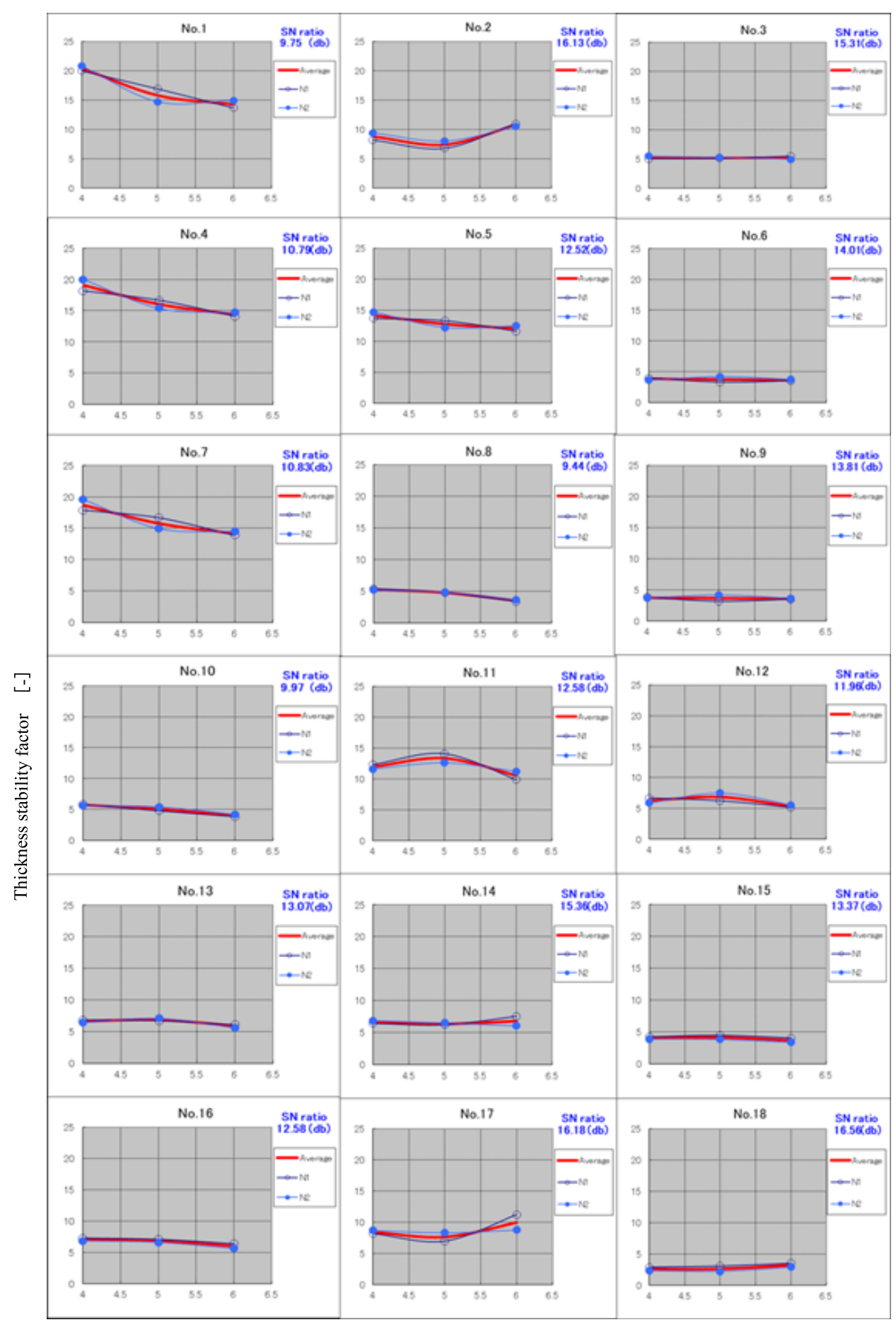

Bonding time $[\mathrm{s}]$

Figure 10: Relationship between bonding time and thickness stability factor.

the adhesion time (horizontal axis), which is the input for each L18 orthogonal table experiment No., and the plate thickness stability factor (vertical axis), which is the output. The graph shows that the relationship between input and output is a characteristic element that evaluates the presence or absence of linearity related to the stability of adhesive strength and plate thickness accuracy.
4.7 Calculation of $\mathrm{SN}$ ratio and slope $\boldsymbol{\beta}$ The characteristic evaluation of the functionality evaluation method uses the SN ratio of dynamic characteristics, and it is ideal that a stable output can be obtained even if there are various noise that affect the functional characteristics. To evaluate the functionality, change the input signal and examine the linearity of the output. This is the SN ratio of the dynamic characteristics. 
Table 5: SN ratio and slope $\beta$.

\begin{tabular}{ccccccccccc}
\hline $\begin{array}{c}\text { EXP. } \\
\text { N0. }\end{array}$ & $\mathrm{A}$ & $\mathrm{B}$ & $\mathrm{C}$ & $\mathrm{D}$ & $\mathrm{E}$ & $\mathrm{F}$ & $\mathrm{G}$ & $\mathrm{H}$ & $\begin{array}{c}\text { SN ratio } \\
(\mathrm{db})\end{array}$ & $\begin{array}{c}\text { Slope } \beta \\
(-)\end{array}$ \\
\hline 1 & 2 & 2.2 & 6.5 & 7 & 8 & 100 & 1.5 & $\mathrm{e} 1$ & 9.75 & 3.20 \\
2 & 2 & 2.2 & 7.5 & 9 & 10 & 120 & 2.5 & $\mathrm{e} 2$ & 16.14 & 1.78 \\
3 & 2 & 2.2 & 8.5 & 11 & 12 & 140 & 3.5 & $\mathrm{e} 3$ & 15.31 & 1.02 \\
4 & 2 & 3.2 & 6.5 & 7 & 10 & 120 & 3.5 & $\mathrm{e} 3$ & 10.79 & 3.15 \\
5 & 2 & 3.2 & 7.5 & 9 & 12 & 140 & 1.5 & $\mathrm{e} 1$ & 12.51 & 2.51 \\
6 & 2 & 3.2 & 8.5 & 11 & 8 & 100 & 2.5 & $\mathrm{e} 2$ & 14.00 & 0.73 \\
7 & 2 & 4.2 & 6.5 & 9 & 8 & 140 & 2.5 & $\mathrm{e} 3$ & 10.83 & 3.11 \\
8 & 2 & 4.2 & 7.5 & 11 & 10 & 100 & 3.5 & $\mathrm{e} 1$ & 9.43 & 0.86 \\
9 & 2 & 4.2 & 8.5 & 7 & 12 & 120 & 1.5 & $\mathrm{e} 2$ & 13.80 & 0.71 \\
10 & 1 & 2.2 & 6.5 & 11 & 12 & 120 & 2.5 & $\mathrm{e} 1$ & 9.97 & 0.94 \\
11 & 1 & 2.2 & 7.5 & 7 & 8 & 140 & 3.5 & $\mathrm{e} 2$ & 12.58 & 2.32 \\
12 & 1 & 2.2 & 8.5 & 9 & 10 & 100 & 1.5 & $\mathrm{e} 3$ & 11.95 & 1.19 \\
13 & 1 & 3.2 & 6.5 & 9 & 12 & 100 & 3.5 & $\mathrm{e} 2$ & 13.06 & 1.25 \\
14 & 1 & 3.2 & 7.5 & 11 & 8 & 120 & 1.5 & $\mathrm{e} 3$ & 15.36 & 1.29 \\
15 & 1 & 3.2 & 8.5 & 7 & 10 & 140 & 2.5 & $\mathrm{e} 1$ & 13.36 & 0.77 \\
16 & 1 & 4.2 & 6.5 & 11 & 10 & 140 & 1.5 & $\mathrm{e} 2$ & 12.57 & 1.28 \\
17 & 1 & 4.2 & 7.5 & 7 & 12 & 100 & 2.5 & $\mathrm{e} 3$ & 16.17 & 1.72 \\
18 & 1 & 4.2 & 8.5 & 9 & 8 & 120 & 3.5 & $\mathrm{e} 1$ & 16.56 & 0.56 \\
\hline
\end{tabular}

Table 6: Factor effect table.

\begin{tabular}{crrrr|rrrr}
\hline & \multicolumn{3}{c}{ SN ratio Total average 13.008 } & \multicolumn{4}{c}{ Slope $\beta$ Total average 1.577 } \\
Factors & Dispersion & Level 1 & Level 2 & Level 3 & Dispersion & Level 1 & Level 2 & Level 3 \\
\hline A & 4.512 & 2.0 & 1.0 & - & 1.832 & 2.0 & 1.0 & - \\
& & -0.501 & 0.501 & - & & 0.319 & -0.319 & - \\
B & 0.693 & 2.2 & 3.2 & 4.2 & 0.21 & 2.2 & 3.2 & 4.2 \\
& & -0.392 & 0.173 & 0.219 & & 0.164 & 0.04 & -0.204 \\
C & 15.627 & 6.5 & 7.5 & 8.5 & 2.767 & 6.5 & 7.5 & 8.5 \\
& & -1.844 & 0.689 & 1.155 & & 0.58 & 0.167 & -0.747 \\
D & 1.130 & 7.0 & 9.0 & 11.0 & 1.487 & 7.0 & 9.0 & 11.0 \\
& & -0.268 & 0.501 & -0.233 & & 0.402 & 0.155 & -0.557 \\
E & 1.944 & 8.0 & 10.0 & 12.0 & 0.411 & 8.0 & 10.0 & 12.0 \\
& & 0.172 & -0.636 & 0.463 & & 0.290 & -0.071 & -0.219 \\
F & 2.941 & 100.0 & 120.0 & 140.0 & 0.307 & 100.0 & 120.0 & 140.0 \\
& & -0.615 & 0.762 & -0.147 & & -0.085 & -0.171 & 0.256 \\
G & 0.856 & 1.5 & 2.5 & 3.5 & 0.065 & 1.5 & 2.5 & 3.5 \\
& & -0.349 & 0.401 & -0.053 & & 0.12 & -0.07 & -0.05 \\
H & 5.359 & $\mathrm{e} 1$ & $\mathrm{e} 2$ & $\mathrm{e} 3$ & 0.531 & $\mathrm{e} 1$ & $\mathrm{e} 2$ & $\mathrm{e}$ \\
& & -1.078 & 0.684 & 0.394 & & -0.102 & -0.233 & 0.335 \\
\hline
\end{tabular}

In this experiment, "Energetic $\mathrm{S} / \mathrm{N}$ ratio based on zeropoint proportionality" is adopted, and Table 5 shows the SN ratio and slope $\beta$ value from the output data. Here, the slope $\beta$ is an index of the degree of variation with respect to the magnitude of the average output slope, and is expressed as a true number.

4.8 Factor effect analysis Figure 11 shows the $\mathrm{SN}$ ratio and slope $\beta$ for each control factor in a factorial effect diagram. The factor effect table (Table 6) shows the influence of a factor or combination of factors on characteristic values, and the diagram showing it is a factor effect diagram. The meaning of this diagram extracts factors that have a large effect on the $\mathrm{SN}$ ratio and do not affect the output slope $\beta$. It can be judged that the level is highly significant in sta- bilizing the DISC adhesive strength and thickness accuracy in the adhesive sizing method. In Fig. 11, the optimum levels are the factors [A1: 2.0], [B3: 4.2], [C3: 8.5], [D2: 9.0], [E3: 12.0], [F2: 120.0], [G2: 2.5], and [H2: e2]. The characteristic values obtained vary with respect to the average value for each level. This variability scale was evaluated in the analysis of variance table (Table 7).

From this table, [A; drying method], [C; heater capacity] and [D; adhesive coating thickness], which are factors with a large dispersion ratio, affect the stability of DISC adhesive strength and plate thickness accuracy. It can be estimated that the factor is high. Table 8 shows the optimal combination of control factor levels to obtain stable DISC adhesive strength and thickness accuracy in the adhesive sizing 

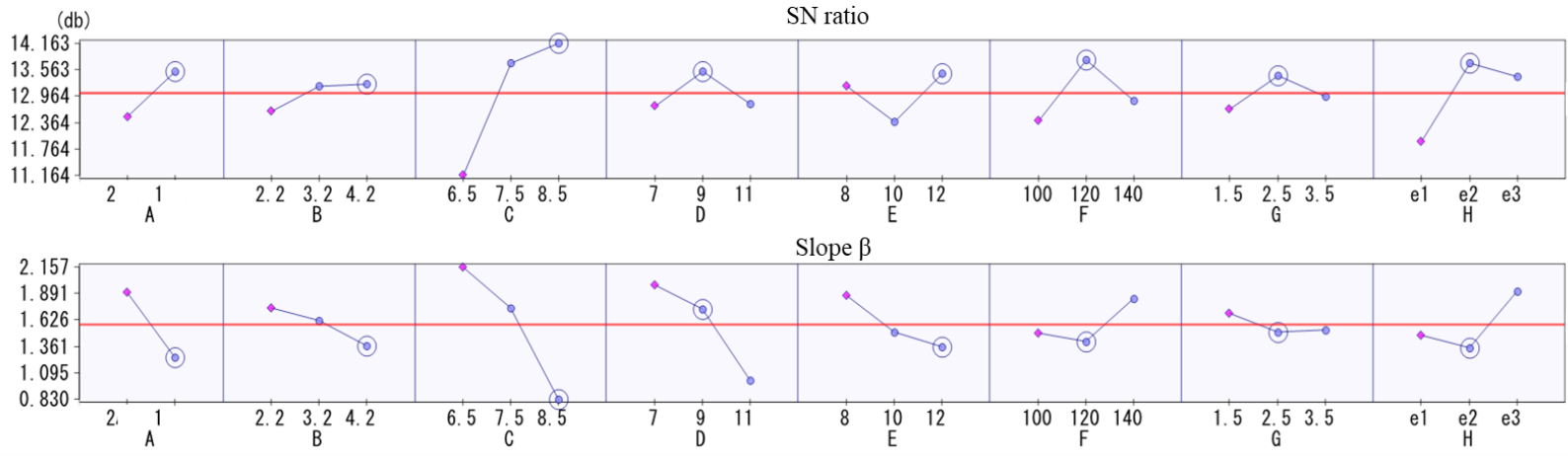

Figure 11: Factor effect diagram.

Table 7: Variance table.

\begin{tabular}{ccccc}
\hline $\begin{array}{l}\text { SN ratio } \\
\text { Factors }\end{array}$ & $\begin{array}{l}\text { Sum } \\
\text { square }\end{array}$ & Freedom & Dispersion & Contribution \\
\hline A & 4.512 & 1 & 4.512 & 0 \\
B & 1.386 & 2 & 0.693 & 0 \\
C & 31.254 & 2 & 15.627 & 4.416 \\
D & 2.26 & 2 & 1.13 & 0 \\
E & 3.888 & 2 & 1.944 & 0 \\
F & 5.881 & 2 & 2.941 & 0 \\
G & 1.712 & 2 & 0.856 & 0 \\
H & 10.718 & 2 & 5.359 & 0 \\
\hline Total & 88.94 & 17 & 5.232 & \\
\hline
\end{tabular}

\begin{tabular}{ccccc}
\hline $\begin{array}{l}\text { Slope } \beta \\
\text { Factors }\end{array}$ & $\begin{array}{l}\text { Sum } \\
\text { square }\end{array}$ & Freedom & Dispersion & Contribution \\
\hline A & 1.832 & 1 & 1.832 & 12.05 \\
B & 0.421 & 2 & 0.21 & 0.5 \\
C & 5.534 & 2 & 2.767 & 37.716 \\
D & 2.973 & 2 & 1.487 & 19.077 \\
E & 0.822 & 2 & 0.411 & 3.419 \\
F & 0.614 & 2 & 0.307 & 1.907 \\
G & 0.131 & 2 & 0.065 & 0 \\
H & 1.061 & 2 & 0.531 & 5.159 \\
\hline Total & 13.74 & 17 & 0.808 & \\
\hline
\end{tabular}

Table 8: Optimization results.

\begin{tabular}{ccccccccccccc}
\hline & & & & & & & & & \multicolumn{2}{c}{ Estimated } & \multicolumn{2}{c}{ Gain } \\
\cline { 9 - 14 } Condition & $\mathrm{A}$ & $\mathrm{B}$ & $\mathrm{C}$ & $\mathrm{D}$ & $\mathrm{E}$ & $\mathrm{F}$ & $\mathrm{G}$ & $\mathrm{H}$ & SN ratio & Slope $\beta$ & SN ratio & Slope $\beta$ \\
\hline Optimal & 1 & 4.2 & 8.5 & 9 & 12 & 120 & 2.5 & $\mathrm{e} 2$ & 17.694 & -0.231 & 9.559 & -3.495 \\
BM & 2 & 2 & 6.5 & 7 & 8 & 100 & 1.5 & $\mathrm{e} 1$ & 8.135 & 3.264 & & \\
\hline
\end{tabular}

method

\subsection{Reliability evaluation of L18 orthogonal table ex-} periment As shown in Table 8, the L18 orthogonal table test results were checked for reliability (additivity) based on the result that the estimated value of the optimum condition was higher than the benchmark condition (BM) and the gain was secured. First, the maximum value and the minimum value of the SN ratio obtained in the L18 orthogonal table experiment are obtained. The judgment method can be judged to be reliable if the difference from the SN ratio of the $\mathrm{BM}$ condition estimated from the value of $10 \%$ of the difference is within $10 \%$ from the statistical rule of thumb [18].

Table 9 shows the reliability evaluation results. Evaluate the dispersion of the proportional relationship between the DISC bonding time and plate thickness stability rate, and it is a desirable condition for stabilizing the DISC bond strength and plate thickness accuracy in the bonding sizing method, it is judged that the L18 orthogonal table experiment is reliable.
Table 9: Reliability of L18 orthogonal experiment.

\begin{tabular}{cccc}
\hline $\begin{array}{c}\text { L18 orthogonal exp. } \\
\text { SN ratio }\end{array}$ & \multicolumn{2}{c}{$\begin{array}{c}\text { By inverse estimation } \\
\text { Reliability check }\end{array}$} \\
\hline Maximum & 16.56 & Obtained from exp. & 9.75 \\
Minimum & 9.43 & SN ratio of BM. & 8.14 \\
Difference & 7.13 & & - \\
\hline 10\% of diff. & $\mathbf{0 . 7 1}$ & $\geq$ & $\mathbf{- 1 . 6 1}$ \\
Judgment & \multicolumn{3}{c}{ Reliable } \\
\hline
\end{tabular}

4.10 Evaluation of reproducibility by confirmation experiment The purpose of the confirmation experiment is to confirm whether the output is stable even when the time, place, and environment change compared to the orthogonal table experiment. Table 10 shows the evaluation results of the confirmation experiment data. In the confirmation experiment, the $\mathrm{SN}$ ratio of the selected optimum condition and BM condition is obtained, and the same two types as in the orthogonal table experiment are performed again to obtain the $\mathrm{SN}$ ratio. By comparing the difference 
Table 10: Reliability of L18 orthogonal experiment.

\begin{tabular}{ccccccccc}
\hline Signal factor & \multicolumn{2}{c}{ M1; 4.0(s) } & \multicolumn{2}{c}{ M2; 5.0(s) } & \multicolumn{2}{c}{ M3; 6.0(s) } & Slope $\beta$ & SN ratio \\
\hline Noise factor & $\mathrm{N} 1$ & $\mathrm{~N} 2$ & $\mathrm{~N} 1$ & $\mathrm{~N} 2$ & $\mathrm{~N} 1$ & $\mathrm{~N} 2$ & $\beta$ & $\eta$ \\
\hline BM & 8.2 & 4.5 & 17.1 & 5.6 & 11.2 & 8.8 & 1.85 & 7.68 \\
Optimal & 18.2 & 13.7 & 19.1 & 14.1 & 18.0 & 19.3 & 3.37 & 16.31 \\
\hline
\end{tabular}
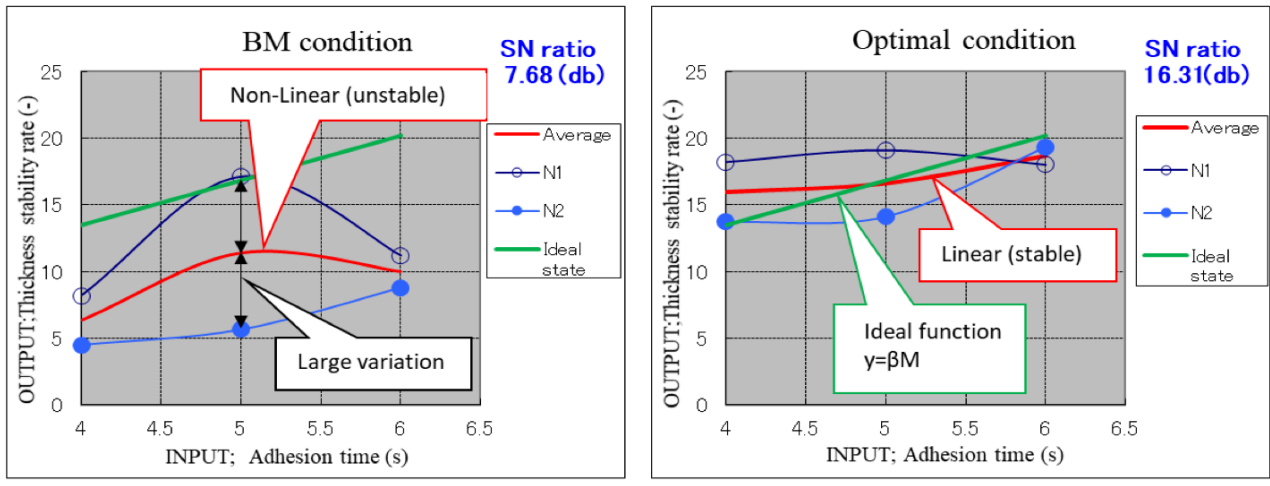

Figure 12: Input/output linearity verification results.

Table 11: Reproducibility of confirmation experiment.

\begin{tabular}{cccc}
\hline \multicolumn{4}{c}{ SN ratio [db] } \\
\hline & Estimated & Confirmated & Reproducibility \\
\hline BM & 8.14 & 7.68 & $94 \%$ \\
Optimal & 17.69 & 16.31 & $92 \%$ \\
\hline Gain & 9.55 & 8.63 & $\mathbf{9 0 \%}$ \\
Repro. & $70 \% \leqq$ & $\underline{\mathbf{9 0 \%}}$ & $\leqq 130 \%$ \\
Gain diffe. & $\underline{\mathbf{0 . 9 2}}$ & $\leqq \pm 3 \mathrm{~dB}$ & \\
\hline Judgment & \multicolumn{4}{c}{ Reproducible } \\
Thickness stability & $\underline{\mathbf{3 9 \%}}$ \\
\hline
\end{tabular}

between the estimated values of the two and the difference (gain) in the confirmation experiment, the reproducibility of the experiment is examined [18].

Table 11 shows the reproducibility evaluation results in the confirmation experiment. In parameter design, if the reproducibility of gain (\%) is within the range of $70 \%$ to $130 \%$, or the gain difference $(\mathrm{db})$ is within $\pm 3 \mathrm{db}$, it can be judged that there is reproducibility. In this experimental result, the reproducibility of the gain was $90 \%$ and the gain difference was $0.92(\mathrm{db})$. It was judged that there was reproducibility. Furthermore, the plate thickness stability with respect to the BM condition was improved by about $40 \%$, and it was shown that the optimum condition extracted was appropriately selected and approached the defined ideal function state.

Figure 12 is a graph comparing the input and output variations and the linear relationship between the optimum and $\mathrm{BM}$ condition in the verification experiment. It shows the change of output (plate thickness stability factor) with respect to input (adhesion time), and it can be seen that the optimal condition is a stable linear proportional relationship with less variation than the BM condition.
4.11 Process capability evaluation by confirmation experiment Table 12 shows the evaluation results of process capability under BM conditions and optimum conditions for the DISC thickness standard $(2.50 \pm 0.03 \mathrm{~mm})$ after adhesion sizing by a confirmation experiment. The measurement data represents the average value of the plate thickness at 6 locations per DISC, and the number of samples is $n=20$ (DISC).

"Process capability" is the probability capability that falls within the product specifications for quality. The "Process Capability Index $\left(C_{p}\right)$ " is used as a measure that shows the degree of variation in quality characteristics due to process variations and the stability with respect to the standard width. $C_{p} \geqq 1.00$ is necessary, and if $C_{p} \geqq 1.33$, it can be determined that the process capability is sufficient. From the results of Table 12, the process capability is insufficient at $C_{p}=0.75<1.33$ under the BM condition, but the process capability is sufficient at $C_{p}=1.53 \geqq 1.33$ under the optimum condition.

$C_{p}$ is calculated by the following formula.

$$
C_{p}=\frac{\mathrm{USL}-\mathrm{LSL}}{6 \sigma}
$$

USL : upper limit specification value LSL : lower limit specification value $\sigma:$ Standard deviation

$$
\sigma=\sqrt{\frac{1}{n} \sum_{n=1}^{n}\left(x_{i}-\bar{x}\right)^{2}}
$$

$n:$ Total number of data

$x_{i}$ : Individual data

$\bar{x}$ : Average value 
Table 12: Process capability index.

\begin{tabular}{ccccc}
\hline \multicolumn{2}{c}{ BM condition } & & \multicolumn{2}{c}{ Optimal condition } \\
Sample & Data & & Sample & Data \\
\cline { 5 - 6 } 1 & 2.540 & & 1 & 2.500 \\
2 & 2.540 & & 2 & 2.510 \\
3 & 2.520 & & 3 & 2.510 \\
4 & 2.530 & & 4 & 2.500 \\
5 & 2.520 & & 5 & 2.495 \\
6 & 2.540 & & 6 & 2.510 \\
7 & 2.505 & & 7 & 2.510 \\
8 & 2.510 & & 8 & 2.500 \\
9 & 2.520 & & 9 & 2.520 \\
10 & 2.510 & & 10 & 2.510 \\
11 & 2.535 & & 11 & 2.500 \\
12 & 2.520 & & 12 & 2.505 \\
13 & 2.510 & & 13 & 2.500 \\
14 & 2.530 & & 14 & 2.500 \\
15 & 2.495 & & 15 & 2.495 \\
16 & 2.520 & & 16 & 2.500 \\
17 & 2.540 & & 17 & 2.510 \\
18 & 2.535 & & 18 & 2.500 \\
19 & 2.535 & & 19 & 2.500 \\
20 & 2.530 & & 20 & 2.510 \\
\cline { 5 - 6 } Average & 2.524 & & Average & 2.504 \\
$\sigma$ & 0.013 & & $\sigma$ & 0.007 \\
$C_{p}$ & 0.75 & & $C_{p}$ & 1.53 \\
STW. & 0.060 & & STW. & 0.060 \\
USL. & 2.530 & & USL. & 2.530 \\
LSL. & 2.470 & & LSL. & 2.470 \\
\hline & & & &
\end{tabular}

\section{Conclusion}

In this study, we dealt with the quality issues of adhesion failure and uneven thickness in DISC adhesion sizing process. In particular, we approached the problem of gear shifting performance and power transmission torque deterioration, which are important functions of DISC, due to $\mathrm{Fa}$ peeling and uneven thickness. Focusing on the correlation between DISC adhesive strength and plate thickness accuracy, we verified the optimization of the processing conditions of the adhesive sizing method using the robust design functionality evaluation method.

As a result of research, we considered the cause system of Fa peeling phenomenon, which is poor adhesion, and applied functional evaluation method. The optimum processing conditions contributing to the stabilization of DISC adhesive strength and plate thickness accuracy could be clarified. In addition, the reproducibility of the optimum conditions obtained was evaluated, and it was confirmed that the plate thickness stability was improved by about $40 \%$ compared to the BM conditions and the process capability was improved by about $50 \%\left(C_{p}=0.75 \rightarrow 1.53\right)$.

Improving automatic transmission performance of automobile automatic transmission is an ongoing technical proposition. In future research, we plan to tackle issues related to the relative thickness accuracy of DISCs installed in multiple transmissions and their effects on gear shifting performance.

\section{References}

[1] Kimura, Y. and Okabe, H., "Tribology outline", YOKENDO, pp.50-110, 1994.

[2] Okamoto, J. and Nakayama K., "Introduction to tribologyfriction, abrasion, the lubricant basics", SAIWAI SHOBO, pp.25-80, 2015.

[3] Haj-Fraj, A. and Pfeiffer, F., "Modeling of Wet Clutches in Automatic Transmissions", The 2nd International Conference on Recent Advances in Mechatronics, pp.9-15, 1999.

[4] Kato, Y. and Shibayama, N., "Structure of the automatic transmission, a function and wet process clutch ", Tribologists, Vol.39, twelfth, pp.6-11, 1994.

[5] Kato, Y. and Akasaka, R., "Friction properties analysis of wet process friction materials for automatic transmission", Tribologists, Vol.39, twelfth, pp.71-78, 1994.

[6] Miura, T. and Sekine, N., "About friction properties of the automotive wet process clutch in the segment adhesion method", HONDA Technical Review, Vol.10, pp.142-149, 2011.

[7] Nakamura, T., "Energy saving of the car by the progress of the tribology technology”, Tribologists, Vol.61, No.2, pp.3108, 2016.

[8] Takahashi, J., "Surface texture of power transmission clutch”, Tribologists, Vol.48, No.9, pp.703-708, 2003.

[9] Sasaki, S., "Improvement of tribological characteristics by surface texturing", Surface technology, Vol.65, No.12, pp.568-572, 2014.

[10] Umehara, T., "Creation of functional surface for improving tribological characteristics", J. Vac. Soc. Jpn., Vol.58, No.6, pp.125-250, 2015.

[11] Koshimizu, S. and Suzuki, M., "Practice Quality Engineering”, Nikkan Kogyo Shimbun, pp.3-56, 2007.

[12] Hirose, K. and Ueda,T., "Introduction to Taguchi Method Analysis Method”, Doyukan, pp.1-24, 2015.

[13] Taguchi, G., "Parameter design in new product development", Japan Standards Association, pp.1-74, 1988.

[14] Taguchi, G., "Quality engineering for technology development”, Japan Standards Association, pp.1-24. 1999.

[15] Suzuki, M., "Quality engineering not difficult", Nikkan Kogyo Shimbun, pp.34-48, 2016.

[16] Yano, H., "Introduction to Quality Engineering Calculation Method", Japan Standards Association, pp.74-137, 2002.

[17] Turuta, M., "Energetic S/N ratio", Nikkagiren, pp.17-72, 2016.

[18] Taguchi, G., "Experimental design and quality engineering", Journal of quality engineering, Vol.2, No.1, pp.2-8,1993. 


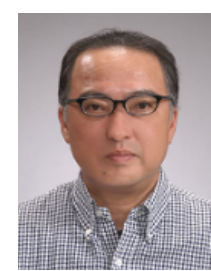

Eiji Toma (Member) was born in Hokkaido, Japan, on December 17, 1959. When he was engaged as a manufacturing engineer at an automobile parts manufacturer, acquired "Professional Engineer (P.E.jp)" in 2010. He is presently professor at National Institute of Technology, Tomakomai College. He obtained P.h.D in 2020 at Hokkaido University of Science. He was engaged in research related to quality engineering practice.

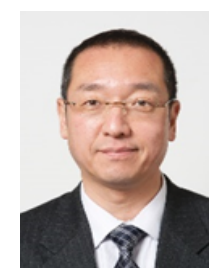

Katsumi Miyama (Non-member) was Graduated from Hokkaido University Department of Metal Engineering in March 1987. Engaged in development work at automobile and electronic parts manufacturers. He obtained "P.E.jp (Metal Division" in 1999 and P.h.D in 2012. He was engaged in research on metal bonding as a professor at Hokkaido University of Science. 